\title{
Expression analysis and clinical significance of CXCL16/CXCR6 in patients with bladder cancer
}

\author{
JUN TAIK LEE ${ }^{1}$, SANG DON LEE ${ }^{2}$, JEONG ZOO LEE ${ }^{2}$, MOON KEE CHUNG $^{2}$ and HONG KOO HA ${ }^{2}$ \\ ${ }^{1}$ Department of Urology, Busan Saint Mary General Hospital; ${ }^{2}$ Department of Urology, \\ Pusan National University School of Medicine, Busan, Republic of Korea
}

Received July 19, 2012; Accepted October 2, 2012

DOI: $10.3892 / \mathrm{ol} .2012 .976$

\begin{abstract}
The interactions between chemokines and their receptors are closely involved in the progression and metastasis of cancer. We hypothesized that the CXCL16-CXCR6 ligandreceptor system plays an important role in bladder cancer progression. To evaluate this hypothesis, the expression levels of CXCL16 and CXCR6 were evaluated in 160 patients, including 155 patients with bladder cancer and 5 patients with benign bladder disease. The tissues were analyzed by immunohistochemical (IHC) staining and real-time reverse-transcription polymerase chain reaction. We compared the expression of CXCL16/CXCR6 in bladder cancer and benign bladder disease. The expression of CXCR6 was increased in patients with bladder cancer compared with benign bladder disease in RT-PCR. The mRNA expression levels of CXCL16 and CXCR6 were $1.75 \times 10^{-2}$ and $1.99 \times 10^{-2}$ in benign bladder tissue and $1.39 \times 10^{-2}$ and $2.32 \times 10^{-2}$ in bladder cancer tissue, respectively. In IHC staining, the expression of CXCL16/CXCR6 in bladder cancer tissues was higher compared with benign bladder tissues. On multivariate analysis, the IHC staining of CXCL16 was correlated with the 2004 WHO grade and lymphovascular invasion $(\mathrm{P}=0.021$ and $\mathrm{P}=0.011$, respectively). CXCR6 was correlated with the 1973 WHO grade $(\mathrm{P}=0.001)$, 2004 WHO grade $(\mathrm{P}<0.001)$, pathological T stage $(\mathrm{P}=0.002)$ and perineural invasion $(\mathrm{P}=0.031)$. However, $\mathrm{Cox}$ regression analysis revealed that the expression of CXCL16 and CXCR6 was not correlated with cancer recurrence and cancer-specific survival ( $\mathrm{P}=0.142$ and $\mathrm{P}=0.324$, respectively). The expression of CXCL16/CXCR6 was higher in bladder cancer compared to benign disease and correlated with aggressive cancer behavior. Based on our results, the CXCL16/CXCR6 axis appears to be important in the progression of bladder cancer. Thus, CXCL16 and CXCR6 serve as potential therapeutic targets.
\end{abstract}

Correspondence to: Professor Hong Koo Ha, Department of Urology, Pusan National University Hospital, 305 Gudeok-Ro, Seo-Ku, Busan, 602739, Republic of Korea

E-mail: hongkooha@naver.com

Key words: bladder, bladder cancer, chemokines, prognosis

\section{Introduction}

Bladder cancer is the fifth most common malignancy in western countries. Incidence increased at a rate of almost 0.8\% per year between 1975 and 1987 and has since leveled off, with an estimated 68,810 new cases in 2008 (1). Despite recent multidisciplinary advances in its treatment, bladder cancer continues to carry unacceptably high rates of mortality and morbidity, with a 10 -year survival rate of $40-50 \%$ (2). More than $70 \%$ of bladder cancers present as moderate- to well-differentiated non-muscle-invasive papillary cancer and are treated with endoscopic transurethral resection. However, $50 \%$ of patients suffer intravesical recurrence within 2 years, and $5-25 \%$ progress to muscle-invasive cancer following repeated recurrence (3). Local recurrence and distant metastasis of tumor cells are not a simple process, but a highly organized phenomenon composed of a series of molecular events. To date, several molecules including chemokines have been identified to play pivotal roles in cancer progression (3). Variable biological behavior in bladder cancer may also be related to differences in expression of chemokines and its receptors (4).

Chemokines are a superfamily of small, secreted proteins (8-10 kDa) and another class of biomarkers that primarily direct the migration of various leukocyte types through interactions with a group of seven-transmembrane-stretch $\mathrm{G}$ protein-coupled receptors resulting in angiogenesis, collagen production, B-cell lymphopoiesis and bone marrow myelopoiesis (5). To date, over 50 chemokines and 20 chemokine receptors have been identified, and are grouped into four categories ( $\mathrm{C}, \mathrm{CC}, \mathrm{CXC}$ and $\mathrm{CX} 3 \mathrm{C})$ according to the location of the main cysteine residues near the $\mathrm{N}$ terminal of these proteins (6). CXCL16 is one of the two known transmembrane chemokines; it is found not only in immune cells, but also expressed constitutively in fibroblasts, keratinocytes and cancer cells of different origins (7-9). In a previous study, CXCL16 was identified as a ligand for CXCR6, which is expressed by peripheral blood leukocytes (10). Eisenhardt et al (4) showed that the chemokine receptor was a noteworthy candidate for the future investigation of metastasis of bladder cancer in vivo. However, there is no study concerning the clinical significance of CXCL16/CXCR6 in bladder cancer.

Toinvestigate whether the CXCL16/CXCR6ligand-receptor system is involved in the progression of human bladder cancer, we performed comparative analyses of immunohistochemical 
(IHC) staining for CXCL16/CXCR6 and quantitative real-time reverse transcription polymerase chain reaction (RT-PCR) in bladder cancer tissues and benign bladder tissues and evaluated the correlation between CXCL16/CXCR6 expression and the clinicopathological findings with reference to characteristics of bladder cancer.

\section{Materials and methods}

Tissue collection. Bladder cancer and benign tissues were obtained from 160 patients. Of these, 155 patients with bladder cancer underwent radical cystoprostatectomy and 5 patients with benign bladder diseases (control group) underwent partial cystectomy or bladder biopsy. Benign bladder lesions included 2 cases with inflammation, 1 with bladder rupture and 2 with pathologically normal bladder. This study was conducted with the approval of Pusan National University clinical trial (PNUH IRB-2011203). Written informed patient consent was obtained from the patients.

The bladder cancer patients were initially evaluated at 1 month after surgery, then every 3 months for 2 years, every 6 months for the next 3 years and every year thereafter until disease progression or mortality. Overall survival (OS) data that reflected overall mortalities were obtained from the Korean National Statistics Registry Database.

IHC staining. We evaluated IHC staining with 155 paraffin blocks and frozen tissues in patients with bladder cancer or benign bladder diseases. The tissues were fixed routinely with formalin and embedded in paraffin. Antigen retrieval was carried out and $0.3 \% \mathrm{H}_{2} \mathrm{O}_{2}$ in phosphate-buffered saline (PBS) was used to block endogenous peroxidase activity in the sections. Following treatment with protein-blocking solution containing $10 \%$ bovine serum albumin (BioShop, Burlington, ON, Canada) in PBS to block non-specific binding, the sections were incubated for $1 \mathrm{~h}$ at room with rabbit anti-human CXCR6 $(1 \mathrm{mg} / \mathrm{ml}$, GeneTex Corporation, Zeeland, MI, USA) or rabbit anti-human CXCL16 $(0.3 \mu \mathrm{g} / \mathrm{ml}$, PeproTech, Rocky Hill, NJ, USA) antibodies. Ensuing incubations were carried out with Cy3-coupled secondary antibodies (Molecular Probes, Eugene, OR, USA). The tissue samples were observed at $x 100$ magnification using a fluorescent microscopy (Axiovert 200, Carl Zeiss, Göttingen, Germany) and images were obtained with AxioVs40 software (version 4.7.2, Carl Zeiss). The immunostaining intensity was scored as no staining (score 0), light red (score 1) and deep red (score 2) at x100 magnification. The percentage of positive cells was calculated as $<5 \%$ (score 0 ), $5-25 \%$ (score 1), $25-50 \%$ (score 2), 50-75\% (score 3) and $>75 \%$ (score 4) at x100 magnification. We defined the IHC staining score as the sum of the intensity and percentage scores.

Total RNA extraction. We evaluated 13 frozen tissues to extract mRNA (8 patients with bladder cancer and 5 with benign bladder diseases). To confirm the expression of mRNA transcripts of CXCR6 and its ligand CXCL16, quantitative real-time RT-PCR was performed. Total RNA was extracted using the QIAzol ${ }^{\mathrm{TM}}$ lysis reagent (Qiagen, Valencia, CA, USA).

Reverse transcription of RNA. The prepared RNA was reverse transcribed into complementary DNA (cDNA) in a volume of $20 \mu \mathrm{l}$ containing $5 \mathrm{X}$ RT reaction buffer plus $0.005 \mathrm{M}$ DTT, $1 \mathrm{mM}$ of each dNTP, 20 units RNase inhibitor, $50 \mu \mathrm{M}$ oligo(dT) primer, $2 \mu \mathrm{g}$ total RNA and 200 units of DiaStar ${ }^{\mathrm{TM}}$ RTase (SolGent, Daejeon, South Korea). The mixture was incubated at $50^{\circ} \mathrm{C}$ for $50 \mathrm{~min}$ and then at $70^{\circ} \mathrm{C}$ for $10 \mathrm{~min}$.

Quantitative real-time RT-PCR. CXCR6 and its ligand CXCL16 mRNA were quantified using commercial LightCycler FastStart DNA Master SYBR-Green I (Roche, Basel, Switzerland) using the LightCycler instrument (Roche Molecular Diagnostics, Pleasanton, CA, USA) for real-time PCR and all subsequent quantification steps, according to the manufacturer's instructions.

The PCR primer pairs used for cDNA amplification were as follows: 5' CTGACTCAGCCAGGCAATGG-3' (sense) and 5'-TGAGTGGACTGCAAGGTGGA-3' (antisense) for human CXCL16; 5'-ATGGCAATGTCTTTAATCTCGACAA-3' (sense) and 5'-TGAAAGCTGGTCATGGCATAGTATT-3' (antisense) for human CXCR6; and 5'-GGGGAGCCAAAA GGGTCATCATCT-3' (sense) and 5'-GAGGGGCCATCC ACAGTCTTCT-3' (antisense) for human $G A P D H$. A typical 20- $\mu$ l one-tube PCR assay contained $1 \mu \mathrm{l}$ cDNA (sample) or serially diluted standard cDNA. PCR amplifications were performed in separate tubes for 50 cycles $\left(10 \mathrm{sec}\right.$ at $95^{\circ} \mathrm{C}$; $5 \mathrm{sec}$ at $57^{\circ} \mathrm{C}$; and $15 \mathrm{sec}$ at $72^{\circ} \mathrm{C}$ ) using PCR master mixtures specific for CXCL16, CXCR6 or the GAPDH housekeeping gene. The GAPDH reaction product served as a control for PCR and as a reference for relative quantification of CXCL16 and CXCR6 mRNA.

The number of PCR cycles required to reach the fluorescence threshold was the cycle threshold $(\mathrm{Ct})$. The $\mathrm{Ct}$ value for each sample was proportional to the log of the initial amount of input cDNA. By plotting the $\mathrm{Ct}$ value of an unknown sample on the standard curve, the amount of target sequence in the sample could be calculated. To normalize the CXCL16 and CXCR6 mRNA expression for sample-to-sample differences in RNA input, RNA quality and reverse transcriptase efficiency, we amplified the GAPDH housekeeping gene. From the standard curve, we derived the calculated amounts of CXCL16, CXCR6 and GAPDH.

Statistical analyses. Statistical analyses were conducted using the SPSS software (version 15.0 for Windows; SPSS, Chicago, IL, USA). Statistical differences in CXCL16 and CXCR6 protein expression between bladder cancer and benign bladder tissue were evaluated using Pearson's Chi-square test. The Pearson coefficient was used to assess the statistical significance of differences between molecular and clinicopathological parameters. Univariate survival analysis was performed according to Kaplan-Meier, and differences in the survival curves were assessed with the log-rank test. Multivariate analysis was performed on all of the parameters that were found to be significant on univariate analysis using the Cox regression model with $\mathrm{P}<0.05$ considered to indicate a statistically significant result.

\section{Results}

mRNA level of CXCL16 and CXCR6 in bladder cancer and control group. The mean mRNA levels of CXCL16 in patients 
Table I. Immunohistochemical staining of CXCL16/CXCR6 according to clinical variables.

\begin{tabular}{|c|c|c|c|c|}
\hline \multirow[b]{2}{*}{ Variable } & \multicolumn{2}{|c|}{ CXCL16 } & \multicolumn{2}{|c|}{ CXCR6 } \\
\hline & Mean expression & P-value & Mean expression & P-value \\
\hline \multicolumn{5}{|l|}{ PNI } \\
\hline Negative & 2.40 & 0.017 & 2.61 & 0.007 \\
\hline Positive & 3.53 & & 3.76 & \\
\hline \multicolumn{5}{|l|}{ Nuclear grade (1973) } \\
\hline 1 and 2 & 2.00 & $<0.001$ & 2.26 & $<0.001$ \\
\hline 3 & 3.44 & & 3.59 & \\
\hline \multicolumn{5}{|l|}{ Nuclear grade (2004) } \\
\hline Low & 1.68 & $<0.001$ & 1.86 & $<0.001$ \\
\hline High & 3.32 & & 3.46 & \\
\hline \multicolumn{5}{|l|}{ T stage } \\
\hline Non-muscle-invasive & 2.27 & 0.003 & 2.50 & 0.019 \\
\hline Muscle-invasive & 3.00 & & 3.08 & \\
\hline \multicolumn{5}{|l|}{ CIS } \\
\hline Negative & 2.32 & $<0.001$ & 2.50 & $<0.001$ \\
\hline Positive & 3.36 & & 3.46 & \\
\hline \multicolumn{5}{|l|}{ LVI } \\
\hline Negative & 2.16 & $<0.001$ & 2.44 & 0.001 \\
\hline Positive & 3.35 & & 3.27 & \\
\hline
\end{tabular}

PNI, perineural invasion; CIS, carcinoma in situ; LVI, lymphovascular invasion.

with bladder cancer and in the control group were $1.39 \times 10^{-2}$ and $1.75 \times 10^{-2}$, respectively. The mean mRNA levels of CXCR6 in the cancer and control groups were $2.32 \times 10^{-2}$ and $1.99 \times 10^{-2}$, respectively. The expression of mRNA levels of CXCR6 was higher in the bladder cancer group than in the control group $(\mathrm{P}=0.033)$, whereas CXCL16 mRNA expression in bladder cancer did not show statistical difference when compared with the control group (Fig. 1).

IHC staining scores in bladder cancer and benign bladder tissues. The positive CXCL16 and CXCR6 immunoreactivity was higher in the bladder cancer group than the control group $(\mathrm{P}=0.021$ and $\mathrm{P}=0.007$, respectively, Fig. 2$)$. The mean IHC staining scores of CXCL16 were 2.59 and 0.71 in the bladder cancer group and the control group, respectively. The mean IHC staining scores of CXCR6 were 2.74 and 0.59 , respectively (Fig. 3).

The mean IHC scores of CXCL16/CXCR6 were 2.40/2.61 and $3.53 / 3.76$ in patients without/with perineural invasion; $2.00 / 2.26$ and $3.44 / 3.59$ in patients with nuclear grade 1 and 2 vs. grade 3 patients; 1.68/1.86 and 3.32/3.46 in low-grade vs. high-grade patients, $2.27 / 2.50$ and $3.00 / 3.08$ in patients with non-muscle-invasive vs. muscle-invasive cancer, $2.32 / 2.50$ and 3.36/3.46 in patients without/with carcinoma in situ, and 2.16/2.44 and 3.35/3.27 in patients without/with lymphovascular invasion, respectively (Table I).

Correlation between IHC staining scores and clinical variables in bladder cancer. The IHC staining score of CXCL16

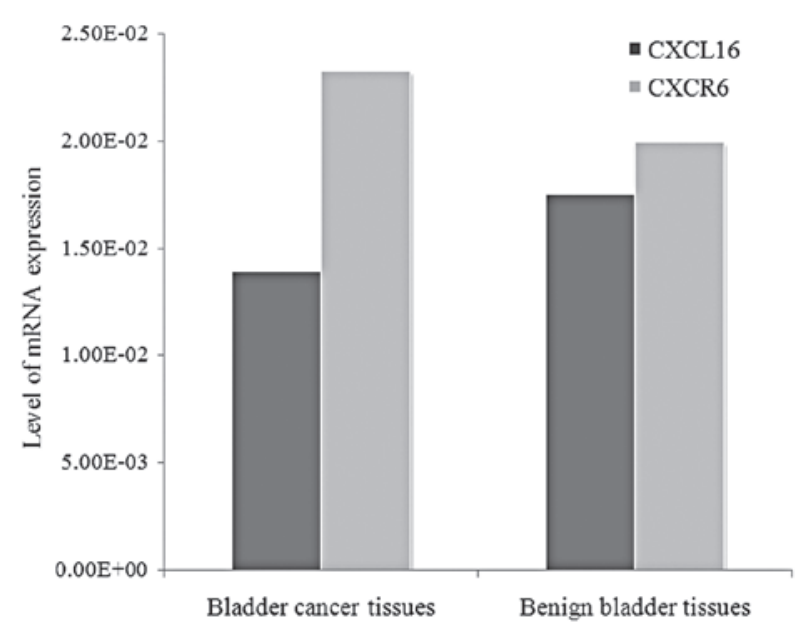

Figure 1. mRNA expression of CXCL16/CXCR6 in bladder cancer and benign bladder tissues.

was correlated with the 1973 WHO grade $(\mathrm{P}=0.008), 2004$ WHO grade $(\mathrm{P}<0.001)$, pathological $\mathrm{T}$ stage $(\mathrm{P}<0.001)$, presence of perineural invasion $(\mathrm{P}=0.008)$, presence of lymphovascular invasion $(\mathrm{P}<0.001)$, presence of positive surgical margin $(\mathrm{P}=0.033)$, presence of ureteral invasion $(\mathrm{P}=0.033)$ and prostatic stromal invasion $(\mathrm{P}<0.001)$. The IHC staining of CXCR6 was correlated with the $1973 \mathrm{WHO}$ grade $(\mathrm{P}<0.001), 2004$ WHO grade $(\mathrm{P}<0.001)$, pathological T stage $(\mathrm{P}=0.001)$, presence of perineural invasion $(\mathrm{P}=0.001)$, presence of lymphovascular invasion $(\mathrm{P}=0.005)$, presence of 

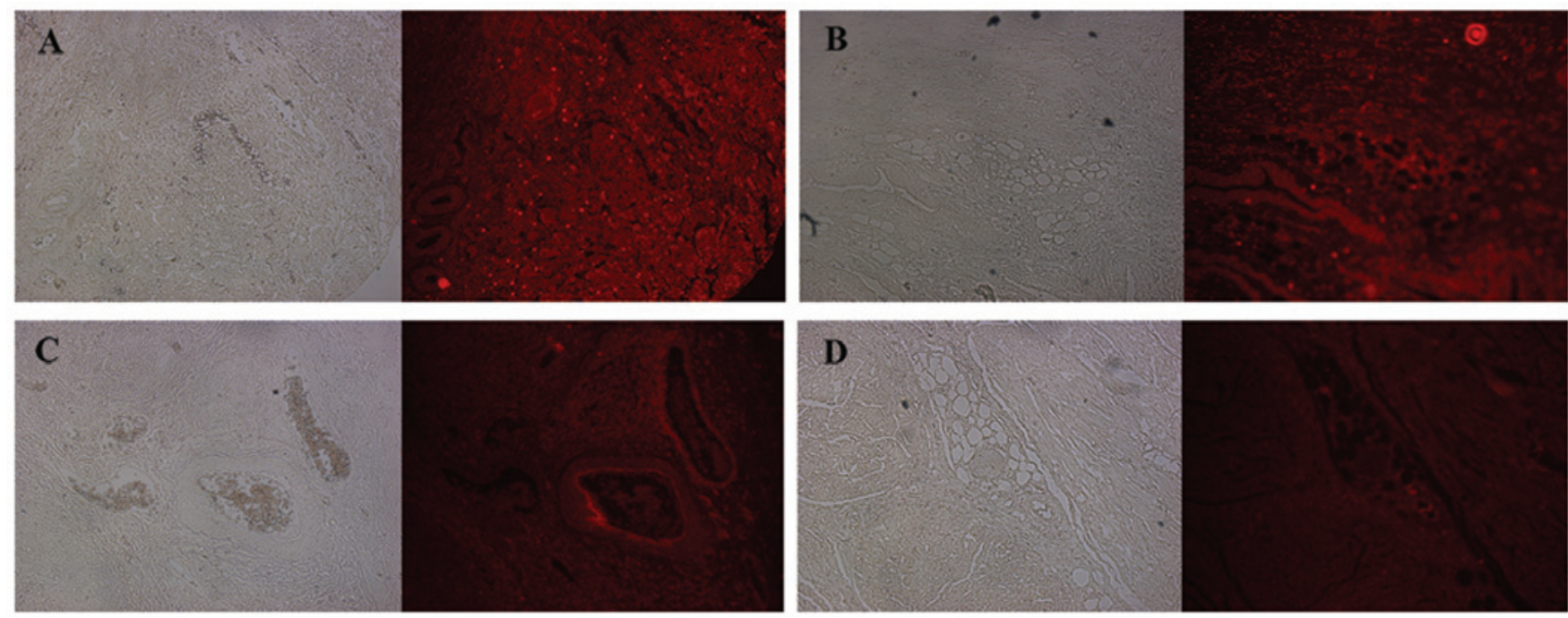

Figure 2. Immunohistochemical staining of CXCL16 and CXCR6 in bladder cancer. (A) High expression of CXCL16, (B) low expression of CXCL16, (C) high expression of CXCR6, (D) low expression of CXCR6.

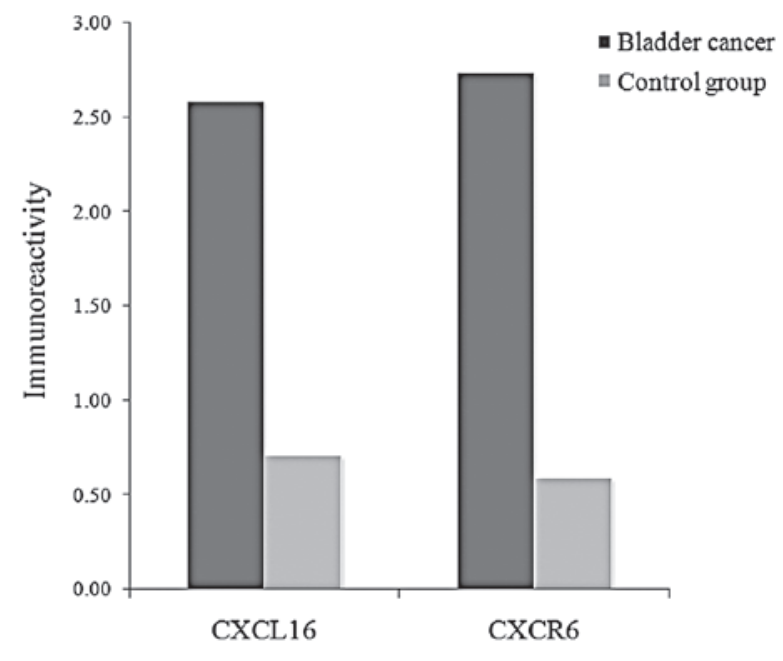

Figure 3. Immunoreactivity of CXCL16/CXCR6 in bladder cancer and benign bladder tissues.

ureteral invasion $(\mathrm{P}<0.001)$, numbers of positive lymph nodes $(\mathrm{P}=0.018)$ and prostatic stromal invasion $(\mathrm{P}=0.001$; Table II $)$.

In multivariate analysis, the IHC staining of CXCL16 was correlated with the 2004 WHO grade and lymphovascular invasion ( $\mathrm{P}=0.021$ and $\mathrm{P}=0.011$, respectively), CXCR6 was correlated with the $1973 \mathrm{WHO}$ grade $(\mathrm{P}=0.001), 2004 \mathrm{WHO}$ grade $(\mathrm{P}<0.001)$, pathological $\mathrm{T}$ stage $(\mathrm{P}=0.002)$ and perineural invasion $(\mathrm{P}=0.031)$.

Prognostic significance of IHC staining scores. There were no significant differences between the IHC staining scores of CXCL16/CXCR6 and 5-year OS $(\mathrm{P}=0.292$ and $\mathrm{P}=0.202$, respectively). For CXCL16, statistical difference between patients with score 0 and 6 could not be obtained since there were very few patients with a score of 0 (4 patients) or 6 (4 patients). However, $50 \%$ of OS showed that higher CXCL16 or CXCR6 IHC staining scores were related to shorter survival duration, which did not show statistical significance.

\section{Discussion}

Transitional cell carcinoma of the bladder is the most frequent tumor of the urinary tract. Despite advances in early detection and therapy, bladder cancer remains a life threatening disease due to the high occurrence of locally advanced or distant metastases. Radical cystectomy is the gold standard treatment for muscle-invasive bladder cancer. However, at least $50 \%$ of patients with locally advanced disease are expected to develop systemic progression within 3 years (2). Patients with advanced bladder cancer are commonly treated with systemic chemotherapy. Analyzing survival rates from standard chemotherapy schedules such as M-VAC (methotrexate, vinblastine, adriamycin and cisplatin) and GC (gemcitabine and cisplatin), results are somewhat discouraging (11). Saxman et al (12) reported that only $5(3.7 \%)$ of 133 of the patients who underwent treatment with M-VAC were alive at the 6-year follow-up duration. This report demonstrated that patients with distant metastases are not cured by systemic chemotherapy. Lehmann et al (13) demonstrated that the GC regimen did not extend overall or progression-free survival more than M-VAC. These reports demonstrated that it is the metastases rather than the primary bladder cancer that cause most cancerrelated mortalities; however, there has been little progress in identifying molecules which were associated metastases. To improve the diagnosis and therapy of localized and metastatic bladder cancer, the identification of molecules involved in the development and progression of this disease is a high priority.

Cancer arises from genetic mutations and epigenetic events in the proliferating cells and changes in the tumor's microenvironment that includes capillaries, smooth muscle cells, fibroblasts and inflammatory cells (14). In 1863, Virchow focused on leukocytes in tumors and suggested that cancer begins at sites associated with chronic inflammation (15). Nonetheless, immunologists have generally considered leukocytes as agents with the potential to limit or eliminate cancers (16). Metastasis is not a simple phenomenon, but a highly complicated process composed of a series of molecular events (17). Several 
Table II. Correlation between immunohistochemical staining of CXCL16/CXCR6 and clinical characteristics.

\begin{tabular}{|c|c|c|c|c|}
\hline Characteristic & No. of patients & Statistical analysis & CXCL16 & CXCR6 \\
\hline 1973 WHO grade & 133 & $\begin{array}{c}\text { Pearson correlation } \\
\text { P-value }\end{array}$ & $\begin{array}{l}0.510 \\
0.008^{\mathrm{a}}\end{array}$ & $\begin{array}{l}0.463 \\
0.000^{\mathrm{b}}\end{array}$ \\
\hline 2004 WHO grade & 90 & $\begin{array}{c}\text { Pearson correlation } \\
\text { P-value }\end{array}$ & $\begin{array}{l}0.485 \\
0.000^{\mathrm{b}}\end{array}$ & $\begin{array}{l}0.482 \\
0.000^{\mathrm{b}}\end{array}$ \\
\hline T stage & 146 & $\begin{array}{c}\text { Pearson correlation } \\
\text { P-value }\end{array}$ & $\begin{array}{l}0.322 \\
0.000^{\mathrm{b}}\end{array}$ & $\begin{array}{l}0.277 \\
0.001^{\mathrm{b}}\end{array}$ \\
\hline CIS & 42 & $\begin{array}{c}\text { Pearson correlation } \\
\text { P-value }\end{array}$ & $\begin{array}{l}0.244 \\
0.119\end{array}$ & $\begin{array}{l}0.229 \\
0.146\end{array}$ \\
\hline PNI & 51 & $\begin{array}{c}\text { Pearson correlation } \\
\text { P-value }\end{array}$ & $\begin{array}{l}0.370 \\
0.008^{\mathrm{a}}\end{array}$ & $\begin{array}{l}0.459 \\
0.001^{b}\end{array}$ \\
\hline LVI & 124 & $\begin{array}{c}\text { Pearson correlation } \\
\text { P-value }\end{array}$ & $\begin{array}{l}0.339 \\
0.000^{\mathrm{b}}\end{array}$ & $\begin{array}{l}0.249 \\
0.005^{\mathrm{a}}\end{array}$ \\
\hline Ureteral invasion & 147 & $\begin{array}{c}\text { Pearson correlation } \\
\text { P-value }\end{array}$ & $\begin{array}{l}0.176 \\
0.033^{\mathrm{a}}\end{array}$ & $\begin{array}{l}0.296 \\
0.000^{\mathrm{b}}\end{array}$ \\
\hline SM & 146 & $\begin{array}{c}\text { Pearson correlation } \\
\text { P-value }\end{array}$ & $\begin{array}{l}0.177 \\
0.033^{\mathrm{a}}\end{array}$ & $\begin{array}{l}0.155 \\
0.062\end{array}$ \\
\hline Positive LN & 101 & $\begin{array}{c}\text { Pearson correlation } \\
\text { P-value }\end{array}$ & $\begin{array}{l}0.159 \\
0.113\end{array}$ & $\begin{array}{l}0.234 \\
0.018^{\mathrm{a}}\end{array}$ \\
\hline Presence of PCA & 88 & $\begin{array}{c}\text { Pearson correlation } \\
\text { P-value }\end{array}$ & $\begin{array}{l}0.395 \\
0.000^{\mathrm{b}}\end{array}$ & $\begin{array}{l}0.346 \\
0.001^{b}\end{array}$ \\
\hline
\end{tabular}

CIS, carcinoma in situ; PNI, perineural invasion; LVI, lymphovascular invasion; SM, surgical margin; LN, lymph node; PCA, prostate cancer. ${ }^{\mathrm{a}} \mathrm{P}<0.05,{ }^{\mathrm{b}} \mathrm{P} \leq 0.001$.

molecular families have been identified to play pivotal roles in cancer metastases. Recently, emerging evidence suggests that a third family, the chemokines and their receptors, are involved in organ-specific metastasis. Chemokines are small, secreted peptides that control the adhesion and trans-endothelial migration of leukocytes, lymphocytes and monocytes, particularly during immune and inflammatory reactions, which were initially researched for their role in the regulation of leukocyte trafficking to inflammatory sites. The chemokine superfamily consists of nearly 50 cytokine members and 20 chemokine receptors $(18,19)$, both involved with enhancing the immunity of tumor-associated antigen, regulating new blood vessel formation, promoting cancer cell proliferation and directing cancer cell metastasis to different destinations. Chemokines and their receptors have also been reported for their significant roles in tumor metastasis, recurrence and angiogenesis in previous studies (20). The interaction of these soluble chemokines with their specific, transmembrane $\mathrm{G}$ protein-coupled receptors mediates their biological effects.

Several recent studies have shown that cancer cells show tumor specific, nonrandom patterns of chemokine receptors and that signaling through these receptors is crucial for chemotactic migration, invasion and metastasis (17). Retz et al (11) showed that bladder cancer cells express CXCR4 progressively with advanced tumorigenesis and that this receptor interacts with CXCL12 to mediate tumor chemotaxis and invasion through connective tissue. These properties identify CXCR4 as a potential target for the attenuation of bladder cancer metastases. During metastasis of prostate cancer CXCR4 protein expression increases in primary and metastatic lesions (21) while the level of CXCL12 was accordingly elevated in the bone (17). There is some indication that a similar correlation may exist between CXCR6 and CXCL16. In fact, Hu et al (22) found that both CXCR6 and CXCR4 are expressed in similar proportions in malignant prostate cancer tissue and benign prostate hyperplasia tissue. In addition, CXCR6 and CXCR4 both show increased expression in malignant tissue. Their corresponding ligands, CXCL16 and CXCL12, were also both found in the human bone tissues. Therefore, it appears both the CXCL12/CXCR4 pathway and CXCL16/CXCR6 pathway are involved in prostate cancer cell migration. CXCL12 and CXCL16 induce migration of prostate cancer cells in an independent extracellular manner. However, this does not preclude the possibility that the two ligands are part of a greater chemokine and chemokine receptor network that mediates cancer cell metastasis and invasion, and that ligands CXCL16 and CXCL12 cooperate or even compete with each other (22). However, there is no study that has investigated the interaction of CXCR6 and its ligand CXCL16 in bladder cancer.

Retz et al (11) demonstrated high mRNA levels of CXCR4 in invasive and locally advanced bladder cancer, and IHC analysis of a case of bladder cancer was consistent with the mRNA expression data for CXCR4. In current study, the expression of mRNA of CXCR6 in bladder cancer tissues was higher than in the control group; however, the expression of mRNA of CXCL16 in bladder cancer tissues was lower than in the control group. This result indicates that malignant bladder tissue itself is more likely associated with CXCR6. 
The significance of the role of chemokines should be determined in a large cohort of bladder tumor patients undergoing long-term observation for disease outcome. Such a study will help determine the prognostic value of mRNA and protein expression of chemokines. In this study, the expression of CXCL16/CXCR6 demonstrated a correlation with survival in bladder cancer patients. Notably, a recent study investigating tissue samples from clear cell renal cell carcinoma found a significant correlation between strong specific chemokine staining and poor tumor-specific survival using multivariate analysis (23). Therefore, monitoring chemokine expression in patients with bladder cancer may improve current tumor staging systems and provide new concepts for adjuvant chemotherapy.

The role for CXCL16/CXCR6 in cancer progression and metastasis has been elucidated through efforts to examine CXCL16 and CXCR6 mRNA in both benign and cancer cell lines in prostate cancer. In a study of prostate cell lines, Ha et al (17) evaluated CXCL16 and CXCR6 mRNA expression both in a benign cell line (PrEC) and in cancer cell lines (LNCaP and PC3). Through real-time PCR, they showed higher concentrations of CXCL16/CXCR6 mRNA levels in the cancer cell lines. However, it remains unknown exactly how CXCL16 and CXCR6 contribute to cancer metastasis and invasion. Lu et al (24) evaluated the role of CXCL16/CXCR6 in cancer aggressiveness. They showed that among prostate cancer cell lines, the more aggressive lines $\mathrm{C} 4-2 \mathrm{~B}$ and $\mathrm{PC} 3$ expressed higher levels of CXCL16/CXCR6 mRNA than the less aggressive LNCaP. Additional studies of actual prostate tissue have similarly supported the existence of a positive correlation between CXCL16/CXCR6 expression and cancer aggressiveness (17). An IHC study by Wang et al (24) demonstrated that CXCR6 expression, which showed strong epithelial staining, was correlated with Gleason score, whereas the expression of CXCL16 was not correlated with Gleason score. Similarly, in the current experiment, the mRNA expression of CXCR6 in the bladder cancer group was higher than that in the control group, whereas CXCL16 was not. Coupled with their previous cell line studies, this result appears to corroborate the results of Ha et al (17) that CXCL16 and total CXCR6 mRNA expression are higher in more aggressive cancer cases. Other recent findings from studies of prostate cancer tissue have provided further confirmation that the CXCL16/CXCR6 expression level is closely associated with high malignant features, as observed with the Gleason grade and tumor stage in prostate cancer (25). Metastatic bone marrow, but not the liver and lung tissues, expressed higher levels of CXCL16 and total CXCR6 mRNA than the primary prostate cancer tissues, implying that the metastatic process was correlated with chemokines and its receptors (17). Soluble CXCL16 induces the migration of CXCR6-expressing cancer cells and enhances the proliferation of these cancer cells with CXCR6 expression in vitro.

Since CXCL16 and CXCR6 are co-expressed in cancer cells, as shown in the present study, it is difficult to discriminate between their individual roles in cancer formation and metastasis. Recent studies have suggested that interaction between CXCR6 and CXCL16 influences angiogenesis (26). Angiogenesis, in turn, is critical to cancer cell proliferation as it increases blood flow to cancer tissue, further promoting cell growth. It has long been documented that many chemokines are responsible for regulating angiogenesis. In particular, angio- genesis is usually enhanced by chemokines positive for the glutamate-leucin-arginine (ELR) tripeptide motif (ELR+) and inhibited by ELR chemokines (27). However, Wang et al (5) found that CXCL16, though an ELR chemokine, promoted angiogenesis in vitro. The authors induced CXCR6 overexpression in PC3 and C4-2B prostate cancer cell lines, and observed increased IL-6 and IL-8 secretion and further angiogenesis. When they suppressed secretion by limiting CXCR6 expression, angiogenesis slowed accordingly. In vivo, significant new blood vessel formations occurred in tumors from C4-2B cells with CXCR6 overexpression, whereas tumors from C4-2B cells with reduced CXCR6 expression showed suppressed angiogenesis. Wang et al (5) also observed a similar role of CXCR6 in the growth and invasion of the actual prostate cancer cells. Using CXCR6 siRNA transfection in vitro, they found that decreased CXCR6 expression resulted in a reduction of invasion of prostate cancer cell lines PC3 and C4-2B stimulated by sCXCL16. Similarly, reduced expression of CXCR6 in cells limited cancer cell invasion. As expected, tumors generated from C4-2B cells with CXCR6 overexpression were significantly larger than those from control cells, while tumor growth was significantly suppressed in tumors established from cells with reduced CXCR6 by CXCR6-siRNA. These data suggest that the interaction between CXCR6 and soluble CXCL16 promotes the proliferation and invasion of cancer cells. Experiments in other cancer tissues have also shown that soluble CXCL16 induces migration and proliferation of cancer cells. These cancer tissues include pancreatic ductal adenocarcinoma cancer (28), schwannomas (29), renal cancer (30) and nasopharyngeal carcinoma (31). In conclusion, the present study revealed that the expression of CXCL16/CXCR6 was correlated with aggressive behaviors of bladder cancer and survival. Based on our results, the CXCL16/CXCR6 axis appears to be important in the progression of bladder cancer and is a potential therapeutic target.

\section{Acknowledgements}

This study was supported by a grant from the Korean Health Technology R\&D Project, Ministry of Health and Welfare, Republic of Korea (A070001), This study was supported by a grant from the National R\&D Program for Cancer Control, Ministry for Health, Welfare and Family affairs, Republic of Korea (0920050).

\section{References}

1. Latini DM, Lerner SP, Wade SW, Lee DW and Quale DZ: Bladder cancer detection, treatment and outcomes: Opportunities and challenges. Urology 75: 334-339, 2009.

2. Stein JP, Lieskovsky G, Cote R, et al: Radical cystectomy in the treatment of invasive bladder cancer: Long-term results in 1,054 patients. J Clin Oncol 19: 666-675, 2001.

3. Nishizawa K, Nishiyama H, Oishi S, et al: Fluorescent imaging of high-grade bladder cancer using a specific antagonist for chemokine receptor CXCR4. Int J Cancer 127: 1180-1187, 2010.

4. Eisenhardt A, Frey U, Tack M, Rosskopf D, Lummen G, Rubben H and Siffert W: Expression analysis and potential functional role of the CXCR4 chemokine receptor in bladder cancer. Eur Urol 47: 111-117, 2005.

5. Wang J, Lu Y, Koch AE, Zhang J and Taichman RS: CXCR6 induces prostate cancer progression by the AKT/mammalian target of rapamycin signaling pathway. Cancer Res 68: 10367-10376, 2008. 
6. Koizumi K, Hojo S, Akashi T, Yasumoto K and Saiki I: Chemokine receptors in cancer metastasis and cancer cellderived chemokines in host immune response. Cancer Sci 98: $1652-1658,2007$.

7. Hojo S, Koizumi K, Tsuneyama K, et al: High-level expression of chemokine CXCL16 by tumor cells correlates with a good prognosis and increased tumor-infiltrating lymphocytes in colorectal cancer. Cancer Res 67: 4725-4731, 2007.

8. Ludwig A, Schulte A, Schnack C, et al: Enhanced expression and shedding of the transmembrane chemokine CXCL16 by reactive astrocytes and glioma cells. J Neurochem 93: 1293-1303, 2005.

9. Scholz F, Schulte A, Adamski F, et al: Constitutive expression and regulated release of the transmembrane chemokine CXCL16 in human and murine skin. J Invest Dermatol 127: 1444-1455, 2007.

10. Tabata S, Kadowaki N, Kitawaki T, et al: Distribution and kinetics of SR-PSOX/CXCL16 and CXCR6 expression on human dendritic cell subsets and CD4+ T cells. J Leukoc Biol 77: 777-786, 2005

11. Retz MM, Sidhu SS, Blaveri E, et al: CXCR4 expression reflects tumor progression and regulates motility of bladder cancer cells. Int J Cancer 114: 182-189, 2005.

12. Saxman SB, Propert KJ, Einhorn LH, et al: Long-term follow-up of a phase iii intergroup study of cisplatin alone or in combination with methotrexate, vinblastine, and doxorubicin in patients with metastatic urothelial carcinoma: A cooperative group study. J Clin Oncol 15: 2564-2569, 1997.

13. Lehmann J, Retz M and Stockle M: Is there standard chemotherapy for metastatic bladder cancer? Quality of life and medical resources utilization based on largest to date randomized trial. Crit Rev Oncol Hematol 47: 171-179, 2003.

14. Coussens LM and Werb Z: Inflammation and cancer. Nature 420 : 860-867, 2002

15. Balkwill $\mathrm{F}$ and Mantovani A: Inflammation and cancer: Back to Virchow? Lancet 357: 539-545, 2001.

16. de Visser KE, Eichten A and Coussens LM: Paradoxical roles of the immune system during cancer development. Nat Rev Cancer 6: 24-37, 2006

17. Ha HK, Lee W, Park HJ, Lee SD, Lee JZ and Chung MK: Clinical significance of CXCL16/CXCR6 expression in patients with prostate cancer. Mol Med Report 4: 419-424, 2011.

18. Luster AD: Chemokines-chemotactic cytokines that mediate inflammation. N Engl J Med 338: 436-445, 1998.
19. Struyf S, Proost P and Van Damme J: Regulation of the immune response by the interaction of chemokines and proteases. Adv Immunol 81: 1-44, 2003.

20. Balkwill F: The significance of cancer cell expression of the chemokine receptor CXCR4. Semin Cancer Biol 14: 171-179, 2004.

21. Sun YX, Wang J, Shelburne CE, et al: Expression of CXCR4 and CXCL12 (SDF-1) in human prostate cancers (PCa) in vivo. J Cell Biochem 89: 462-473, 2003.

22. Hu W, Zhen X, Xiong B, Wang B, Zhang W and Zhou W: CXCR6 is expressed in human prostate cancer in vivo and is involved in the in vitro invasion of PC3 and LNCap cells. Cancer Sci 99: 1362-1369, 2008.

23. Staller P, Sulitkova J, Lisztwan J, Moch H, Oakeley EJ and Krek W: Chemokine receptor CXCR4 downregulated by von Hippel-Lindau tumour suppressor pVHL. Nature 425: 307-311, 2003.

24. Lu Y, Wang J, Xu Y, et al: CXCL16 functions as a novel chemotactic factor for prostate cancer cells in vitro. Mol Cancer Res 6: 546-554, 2008.

25. Darash-Yahana M,Gillespie JW,Hewitt SM, et al: The chemokine CXCL16 and its receptor, CXCR6, as markers and promoters of inflammation-associated cancers. PLoS One 4: e6695, 2009.

26. Deng L, Chen N, Li Y, Zheng H and Lei Q: CXCR6/CXCL16 functions as a regulator in metastasis and progression of cancer. Biochim Biophys Acta 1806: 42-49, 2010.

27. Belperio JA, Keane MP, Arenberg DA, et al: CXC chemokines in angiogenesis. J Leukoc Biol 68: 1-8, 2000.

28. Gaida MM, Gunther F, Wagner C, et al: Expression of the CXCR6 on polymorphonuclear neutrophils in pancreatic carcinoma and in acute, localized bacterial infections. Clin Exp Immunol 154: 216-223, 2008.

29. Held-Feindt J, Rehmke B, Mentlein R, et al: Overexpression of CXCL16 and its receptor CXCR6/bonzo promotes growth of human schwannomas. Glia 56: 764-774, 2008.

30. Gutwein P, Schramme A, Sinke N, et al: Tumoural CXCL16 expression is a novel prognostic marker of longer survival times in renal cell cancer patients. Eur J Cancer 45: 478-489, 2009.

31. Ou DL, Chen CL, Lin SB, Hsu CH and Lin LI: Chemokine receptor expression profiles in nasopharyngeal carcinoma and their association with metastasis and radiotherapy. J Pathol 210: 363-373, 2006. 